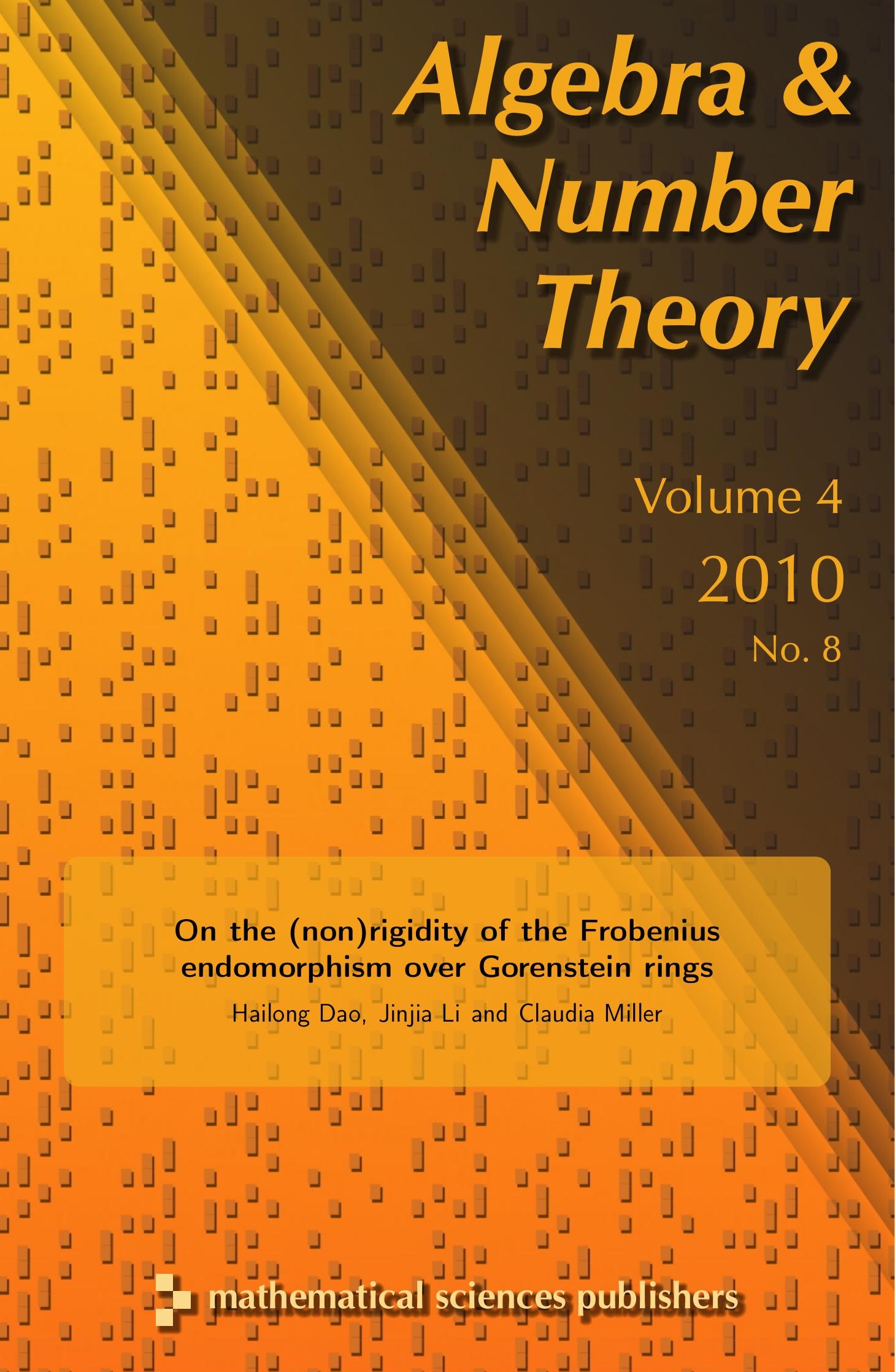

On the (non)rigidity of the Frobenius endomorphism over Gorenstein rings

Hailong Dao, Jinjia Li and Claudia Miller

\lrcorner 


\title{
On the (non)rigidity of the Frobenius endomorphism over Gorenstein rings
}

\author{
Hailong Dao, Jinjia Li and Claudia Miller
}

\begin{abstract}
It is well-known that for a large class of local rings of positive characteristic, including complete intersection rings, the Frobenius endomorphism can be used as a test for finite projective dimension. In this paper, we exploit this property to study the structure of such rings. One of our results states that the Picard group of the punctured spectrum of such a ring $R$ cannot have $p$-torsion. When $R$ is a local complete intersection, this recovers (with a purely local algebra proof) an analogous statement for complete intersections in projective spaces first given by Deligne in SGA and also a special case of a conjecture by Gabber. Our method also leads to many simply constructed examples where rigidity for the Frobenius endomorphism does not hold, even when the rings are Gorenstein with isolated singularity. This is in stark contrast to the situation for complete intersection rings. A related length criterion for modules of finite length and finite projective dimension is discussed towards the end.
\end{abstract}

\section{Introduction}

The Frobenius endomorphism for rings of positive characteristic has been one of the central objects of study in homological commutative algebra over the past decades. Not only is it a useful tool in proofs of homological conjectures, but also its intrinsic homological properties have been shown to have strong connections with the structure of the ring or of modules over it. In this article we provide several surprising connections, for example, the relationship between the ability of the Frobenius to detect the finite projective dimension of modules and the torsion part of the divisor class group.

We review some history and notation. In [Kunz 1969, Theorem 2.1] regular local rings are characterized as those for which the Frobenius endomorphism $f: R \rightarrow R$ (or equivalently some iteration of it) is flat. Since then, a list of papers has yielded further similar homological results for $f$, each analogous to a classical homological

Miller gratefully acknowledges partial financial support from NSA grant \#H98230-06-1-0035. Dao is partially supported by NSF grant 0834050 .

MSC2000: primary 13A35; secondary 13D07, 14A05, 13C20.

Keywords: Frobenius endomorphism, rigidity, Tor, Picard group, isolated singularity. 
result concerning the residue field $k$ (viewed as an $R$-module via $\pi: R \rightarrow k$ ); for further details see the survey [Miller 2003], as well as [Avramov et al. 2006; Iyengar and Sather-Wagstaff 2004]. We will use the notation ${ }^{n} R$ for $R$ viewed as an $R$-module via the $n$-th iteration $f^{n}$ of $f$.

For their celebrated proof of the Intersection Theorem, Peskine and Szpiro [1969, Corollary 2; 1972, Theorem 1.7] generalized one direction of Kunz's result, and shortly thereafter Herzog [1974, Theorem 3.1] proved the converse, yielding the following equivalence:

$M$ has finite projective dimension $\Leftrightarrow \operatorname{Tor}_{i}^{R}\left(M,{ }^{n} R\right)=0$ for all $i>0$ and all $n>0$.

This leads one to ask to what extent the module $f^{n} R$ could function as a test module for finite projective dimension: is the vanishing of $\operatorname{Tor}_{i}^{R}\left(M,{ }^{n} R\right)$ for just one value each of $i>0$ and $n>0$ sufficient? In particular, this would imply that the $R$-module $f^{n} R$ is rigid, that is, that

$$
\operatorname{Tor}_{i}^{R}\left(M, f^{n} R\right)=0 \Longrightarrow \operatorname{Tor}_{i+1}^{R}\left(M,{ }^{n} R\right)=0 .
$$

Several steps toward these goals have been made in recent years. In the general setting, [Koh and Lee 1998, Proposition 2.6] proved a finiteness result: there is a constant $c(R)$, depending only on the ring $R$, such that vanishing of $\operatorname{Tor}_{i}^{R}\left(M,{ }^{f^{n}} R\right)$ for any depth $R+1$ consecutive values of $i>0$ and any one value of $n \geq c(R)$ implies that $M$ has finite projective dimension. In fact, it showed that depth $R$ consecutive values of $i$ suffice if $R$ is Cohen-Macaulay of positive dimension. The best possible result however, occurs in the setting of complete intersection rings:

Theorem 1.1 [Avramov and Miller 2001; Dutta 2003]. Let $R$ be a local complete intersection and $M$ a finitely generated $R$-module. Then the vanishing of $\operatorname{Tor}_{i}^{R}\left(M,{ }^{n} R\right)$ for one value each of $i>0$ and $n>0$ implies that $M$ has finite projective dimension.

Phenomena like this can occur over noncomplete intersection rings as well. In such a case, we call the corresponding ${ }^{f^{n}} R$ strongly rigid (which is equivalent to being rigid when $n \geq c(R)$ by Koh and Lee's result above). See Definition 2.1 and Example 2.2 for known examples.

In Section 2, we study the properties of Gorenstein local rings whose corresponding ${ }^{f^{n}} R$ is strongly rigid. We show that if $R$ is Gorenstein such that ${ }^{n} R$ is locally strongly rigid (i.e., strongly rigid at the localization at every prime ideal), then the minimal infinite projective dimension locus of a module $M$ (see Definition 2.3) must be contained in the set of associated primes of $F^{n}(M)$ (see Theorem 2.5). One consequence of this result is the following characterization for modules of finite projective dimension: 
Corollary 2.6. Let $R$ be a Gorenstein local ring such that ${ }^{f^{n}} R$ is locally strongly rigid for some $n>0$ and $M$ an $R$-module. Then $M$ has finite projective dimension if and only if Ass $F^{n}(M)$ is contained in the finite projective dimension locus of $M$.

Note that the class of rings such that ${ }^{n} R$ is locally strongly rigid for all $n>0$ includes, but is strictly bigger than, the class of all local complete intersections; see Example 2.2.

We also apply Theorem 2.5 to prove that the divisor class groups of certain Gorenstein domains have no $p$-torsion.

Theorem 2.9. Let $R$ be a Gorenstein local ring such that $f^{n} R$ is locally strongly rigid for some $n>0$. Let $I$ be a reflexive ideal such that $I$ is locally free in codimension 2. Furthermore, assume that $\operatorname{Hom}_{R}(I, I) \cong R$. Let $q=p^{n}$. Then if $I^{(q)}$ satisfies Serre's condition $\left(S_{3}\right)$, I must be principal. In particular, the Picard group of the punctured spectrum of $R$ has no $p$-torsion. If, furthermore, $R$ satisfies condition $\left(R_{2}\right), \mathrm{Cl}(R)$ has no p-torsion.

This theorem shows that the Picard groups of the punctured spectrum of complete intersection rings cannot have $p$-torsion. For complete intersections in projective spaces, such a result was first proved in [Deligne 1973, Theorem 1.8] using sophisticated geometric machinery. We also note that this particular case confirms the positive characteristic case of the following conjecture:

Conjecture 1.2 [Gabber 2004]. Let $(R, \mathfrak{m})$ be a local complete intersection ring of dimension 3. Let $U_{R}=\operatorname{Spec}(R)-\{\mathfrak{m}\}$ be the punctured spectrum of $R$. Then $\operatorname{Pic}\left(U_{R}\right)$ is torsion-free.

It was implied in [Gabber 2004] that the positive characteristic case is known, but we cannot find a precise reference. In any case, it is worth noting that our proof is purely homological and quite simple.

In Section 3, we push the ideas in the previous section further to construct many examples of Gorenstein local rings $R$ such that ${ }^{n} R$ is not strongly rigid. In other words, the vanishing of $\operatorname{Tor}_{i}^{R}\left(M,{ }^{n} R\right)$ for just one value each of $i>0$ and $n>0$ is not sufficient to conclude that $M$ has finite projective dimension. Two different approaches are used in these constructions. The first approach boils down to finding an isolated Gorenstein singularity with torsion class group and applying Theorem 2.9; see Example 3.2. The second approach takes a different route, via Lemma 3.3. Obtaining an actual example requires some explicit computations on the determinantal ring of $2 \times 2$ minors in 9 variables and hence is less general than the first approach; see Example 3.5. The bonus is, however, that these have a torsion-free class group.

In Section 4, we study the connection between (strong) rigidity and numerical rigidity (see Definitions 4.1 and 4.2) of the Frobenius endomorphism. The main result we prove there is this: 
Theorem 4.6. Let $R$ be a Cohen-Macaulay local ring with isolated singularity and of positive dimension. Fix $n>0$. If $f^{n}(R / y R)$ is numerically rigid for every nonzerodivisor $y \in R$, then ${ }^{f^{n}} R$ is strongly rigid against modules of dimension up to one.

The rest of the introduction contains a review of the notation and definitions used throughout the paper. We assume throughout that $R$ is a commutative Noetherian local ring of prime characteristic $p>0$ and that all $R$-modules $M$ and $N$ are finitely generated. The Frobenius endomorphism $f: R \rightarrow R$ is defined by $f(r)=r^{p}$ for $r \in R$; its self-compositions are given by $f^{n}(r)=r^{p^{n}}$. Restriction of scalars along each iteration $f^{n}$ endows $R$ with a new $R$-module structure, denoted by $f^{n} R$.

The Frobenius functor, introduced in [Peskine and Szpiro 1972], is given by base change along the Frobenius endomorphism:

$$
F_{R}(M)=M \otimes_{R}{ }^{f} R
$$

for any $R$-module $M$. Its compositions are given by $F_{R}^{n}(M)=M \otimes_{R} f^{n} R$, namely base change along the compositions $f^{n}$ of $f$. We omit the subscript $R$ if there is no ambiguity about $R$. Note particularly that the module structure on $F^{n}(M)$ is via usual multiplication in $R$ on the right hand factor of the tensor product. The values of the derived functors $\operatorname{Tor}_{i}^{R}\left(M, f^{n} R\right)$ are similarly viewed as $R$-modules via the target of the base change map $f^{n}$.

It is easy to verify that $F^{n}(R) \cong R$ and that for cyclic modules $F^{n}(R / I) \cong R / I^{[q]}$, where $q=p^{n}$ and $I^{[q]}$ denotes the ideal generated by the $q$-th powers of the generators of $I$. We frequently use $q$ to denote the power $p^{n}$, which may vary.

In the sequel, $\ell(M)$ will denote the length and $\mathrm{pd} M$ the projective dimension of the module $M$. By the codimension of $M$ we mean $\operatorname{dim} R-\operatorname{dim} M$. We use the notation $\boldsymbol{x}$ for a sequence of elements of $R$ and often write simply $R / \boldsymbol{x}$ for $R /(\boldsymbol{x})$ to save space. Likewise, $x^{q}$ denotes the ideal generated by the $q$-th powers of the sequence $\boldsymbol{x}$, not the $q$-th power of the ideal $\boldsymbol{x}$.

\section{Strong rigidity of Frobenius and torsion elements in divisor class groups}

We now investigate the consequences of the phenomenon that over certain rings the Frobenius map can be used to test for finite projective dimension (e.g., over complete intersection rings). This work enables us to prove strong results about torsion elements in the class groups of complete intersection rings and also allows us to construct counterexamples to such phenomena over noncomplete intersection rings. We begin with some convenient definitions to facilitate the discussion.

Definition 2.1. An $R$-module $N$ is called strongly rigid if for any integer $i$ and any finitely generated $R$-module $M$, $\operatorname{Tor}_{i}^{R}(M, N)=0$ implies $\operatorname{pd}_{R} M<\infty$. The module $N$ is called locally strongly rigid if $N_{\mathfrak{p}}$ is strongly rigid for all $\mathfrak{p} \in \operatorname{Spec} R$. 
Example 2.2. If $R$ is a local complete intersection ring, then ${ }^{n} R$ is locally strongly rigid for all $n$; see Theorem 1.1. For any local Cohen-Macaulay ring $R$ of dimension at most 1 , there is a number $c(R)$ such that for any $n \geq c(R),{ }^{n} R$ is strongly rigid by virtue of [Koh and Lee 1998, Proposition 2.6], already mentioned on page 1040 . In particular, when $(R, \mathfrak{m})$ is Artinian and $\mathfrak{m}^{[p]}=0$, then ${ }^{n} R$ is (locally) strongly rigid for all $n$ [Miller 2003, 2.2.8].

Definition 2.3 [Dao 2010]. Let $M$ be an $R$-module. One defines the infinite projective dimension locus of $M$ as

$$
\operatorname{IPD}(M)=\left\{\mathfrak{p} \in \operatorname{Spec} R \mid \operatorname{pd}_{R_{\mathfrak{p}}} M_{\mathfrak{p}}=\infty\right\} .
$$

Similarly, define $\operatorname{FPD}(M)$ to be the finite projective dimension locus of $M$. Finally, we define the $n$-strong rigidity locus of $R$ as

$$
\operatorname{SR}_{n}(R)=\left\{\mathfrak{p} \in \operatorname{Spec} R \mid{ }^{n} R_{\mathfrak{p}} \text { is strongly rigid }\right\} .
$$

The following standard facts, which we state without proof, will be used often:

Fact 2.4. Let $f: R \rightarrow S$ be a ring homomorphism and $\mathfrak{p}$ a prime ideal of $S$. Then for each $i \geq 0$ and $R$-module $M$ there is a natural isomorphism

$$
\operatorname{Tor}_{i}^{R}(M, S)_{\mathfrak{p}} \cong \operatorname{Tor}_{i}^{R_{f^{-1}(\mathfrak{p})}}\left(M_{f^{-1}(\mathfrak{p})}, S_{\mathfrak{p}}\right) .
$$

Furthermore, if $f$ is the Frobenius endomorphism of $R$, then $f^{-1}(\mathfrak{p})=\mathfrak{p}$ and $R_{f^{-1}(\mathfrak{p})} \rightarrow S_{\mathfrak{p}}$ is the Frobenius endomorphism of $R_{\mathfrak{p}}$.

Theorem 2.5. Let $R$ be a Gorenstein local ring and $M$ an $R$-module. Then

$$
\min \operatorname{IPD}(M) \cap \operatorname{SR}_{n}(R) \subseteq \operatorname{Ass} F^{n}(M) .
$$

In particular, if ${ }^{f^{n}} R$ is locally strongly rigid, then

$$
\min \operatorname{IPD}(M) \subseteq \operatorname{Ass} F^{n}(M) .
$$

Proof. Since $R$ is Gorenstein, by the Cohen-Macaulay approximation due to [Auslander and Buchweitz 1989, 1.8], there is a short exact sequence

$$
0 \rightarrow M \rightarrow Q \rightarrow N \rightarrow 0
$$

where pd $Q<\infty$ and $N$ is maximal Cohen-Macaulay. Tensoring with the Frobenius endomorphism, we have an embedding

$$
0 \rightarrow \operatorname{Tor}_{1}^{R}\left(N, f^{n} R\right) \rightarrow F^{n}(M) .
$$

Take any $\mathfrak{p} \in \min \operatorname{IPD}(M) \cap \mathrm{SR}_{n}(R)$; then $\operatorname{pd}_{R_{\mathfrak{p}}} M_{\mathfrak{p}}=\infty_{R}$ and $f^{n} R_{\mathfrak{p}}$ is strongly rigid. It follows that $\operatorname{pd}_{R_{\mathfrak{p}}} N_{\mathfrak{p}}=\infty$ and therefore that $\operatorname{Tor}_{1}{ }^{R_{\mathfrak{p}}}\left(N_{\mathfrak{p}},{ }^{n} R_{\mathfrak{p}}\right) \neq 0$. On the other hand, since $\mathfrak{p}$ is minimal in the infinite projective dimension locus of $M$, 
$\operatorname{pd}_{R_{\mathfrak{q}}} M_{\mathfrak{q}}<\infty$ for any prime $\mathfrak{q} \subsetneq \mathfrak{p}$, whence $\operatorname{pd}_{R_{\mathfrak{q}}} N_{\mathfrak{q}}<\infty$ and so $\operatorname{Tor}_{1}^{R_{\mathfrak{q}}}\left(N_{\mathfrak{q}}, f^{n} R_{\mathfrak{q}}\right)=$ 0 . Therefore, the length of $\operatorname{Tor}_{1}^{R_{\mathfrak{p}}}\left(N_{\mathfrak{p}},{ }^{n} R_{\mathfrak{p}}\right)$ must be finite.

Localizing (2-1) at $\mathfrak{p}$, we have an exact sequence

$$
0 \rightarrow \operatorname{Tor}_{1}^{R_{\mathfrak{p}}}\left(N_{\mathfrak{p}},{ }^{n} R_{\mathfrak{p}}\right) \rightarrow F^{n}(M)_{\mathfrak{p}}
$$

This implies that depth $F^{n}(M)_{\mathfrak{p}}=0$. Hence $\mathfrak{p} \in \operatorname{Ass} F^{n}(M)$.

Corollary 2.6. Let $R$ be a Gorenstein local ring such that ${ }^{f^{n}} R$ is locally strongly rigid for some $n>0$ and $M$ an $R$-module. Then $M$ has finite projective dimension if and only if $\operatorname{Ass} F^{n}(M) \subseteq \operatorname{FPD}(M)$.

As an immediate consequence, we obtain the following special case with simpler hypotheses. Here, $\operatorname{Sing}(R)$ denotes the singular locus of $R$. Note particularly that the hypothesis that $\min \operatorname{Supp} M$ and $\operatorname{Sing}(R)$ are disjoint holds, for example, when $\operatorname{dim} M>\operatorname{dim} \operatorname{Sing}(R)$.

Corollary 2.7. Let $R$ be a Gorenstein local ring such that ${ }^{f^{n}} R$ is locally strongly rigid for some $n>0$ (e.g., if $R$ is a local complete intersection) and $M$ an $R$-module such that $(\min \operatorname{Supp} M) \cap \operatorname{Sing}(R)=\varnothing$. If $F^{n}(M)$ has no embedded primes, then $M$ has finite projective dimension. In particular, if $F^{n}(M)$ is Cohen-Macaulay, then $M$ is perfect.

Proof. It suffices to note that

$$
\text { Ass } F^{n}(M)=\min \operatorname{Supp} F^{n}(M)=\min \operatorname{Supp} M \subseteq \operatorname{Spec} R \backslash \operatorname{Sing}(R) \subseteq \operatorname{FPD}(M),
$$

where the first equality is by the assumption that $F^{n}(M)$ has no embedded primes, the second is well-known (see [Peskine and Szpiro 1972], for example) and the first containment follows from the hypothesis.

Remark 2.8. If $R$ is reduced, we do not know if the disjointness of min Supp $M$ and $\operatorname{Sing}(R)$ in Corollary 2.7 can be replaced by the simpler condition $\operatorname{dim} M>0$. However, this is impossible when $R$ is not reduced (see [Miller 2003, 2.1.7], for example).

We now give an application of Theorem 2.5 to divisor class groups. In the sequel, we use $\mathrm{Cl}(R)$ to denote the divisor class group of $R$. We refer to [Fossum 1973] for the definition and basic facts about $\mathrm{Cl}(R)$ and the Picard groups and to [Bruns and Herzog 1993] for Serre's conditions $\left(R_{n}\right)$ and $\left(S_{n}\right)$.

Theorem 2.9. Let $R$ be a Gorenstein local ring such that ${ }^{n} R$ is locally strongly rigid for some $n>0$. Let $I$ be a reflexive ideal such that $I$ is locally free in codimension 2. Furthermore, assume that $\operatorname{Hom}_{R}(I, I) \cong R$. Let $q=p^{n}$. Then if $I^{(q)}$ satisfies Serre's condition $\left(S_{3}\right), I$ must be principal. In particular, the Picard group of the punctured spectrum of $R$ has no p-torsion. If, furthermore, $R$ satisfies condition $\left(R_{2}\right)$, then $\mathrm{Cl}(R)$ has no p-torsion. 
Proof. We may assume $\operatorname{dim} R \geq 3$. Assume that $I$ is not principal, then it follows that pd $I=\infty$ (see [Braun 2004, Corollary 11] and [Bourbaki 1965, Chapter VII, $\S 4$, no. 7, Corollary 2]). We claim that one can always write $I=(a):(b)$ for $a, b \in R$. Here is a quick proof: choose $a$ such that $a$ generates $I$ at the minimal primes of $I$. Pick an irredundant primary decomposition of $(a)$; it can be written as $I \cap J$ (if $I=(a)$ we are done). Choosing $b$ in $J$ but not in any minimal prime of $I$, one can show that $I=(a):(b)$. By the short exact sequence

$$
0 \rightarrow R /(a: b) \stackrel{b}{\rightarrow} R /(a) \rightarrow R /(a, b) \rightarrow 0
$$

we obtain $\operatorname{IPD}(I)=\operatorname{IPD}(R /(a, b))$. Thus we have $\mathfrak{p} \in \min \operatorname{IPD}(R /(a, b))$ for any $\mathfrak{p} \in \min \operatorname{IPD}(I)$, and so by Theorem 2.5 ,

$$
\mathfrak{p} \in \operatorname{Ass}\left(F^{n}(R /(a, b))\right)=\operatorname{Ass}\left(R /\left(a^{q}, b^{q}\right)\right) .
$$

Localize the short exact sequence

$$
0 \rightarrow R /\left(a^{q}: b^{q}\right) \rightarrow R /\left(a^{q}\right) \rightarrow R /\left(a^{q}, b^{q}\right) \rightarrow 0
$$

at $\mathfrak{p}$, and observe that $\left(a^{q}: b^{q}\right)=I^{(q)}$. From the fact that $\operatorname{depth}\left(R /\left(a^{q}, b^{q}\right)\right)_{\mathfrak{p}}=0$ we get depth $I_{\mathfrak{p}}^{(q)} \leq 2$. On the other hand, since $I$ is locally free in codimension 2, $\operatorname{dim} R_{\mathfrak{p}} \geq 3$. So, $I^{(q)}$ does not satisfy $\left(S_{3}\right)$, and our first assertion is proved. The last two statements follow immediately (note that if $R$ is $\left(R_{2}\right)$ then $R$ is automatically normal).

As a corollary we can recover a notable result about torsion elements in the Picard groups of complete intersections.

Corollary 2.10. Let $R$ be an equicharacteristic local complete intersection ring of dimension at least 3. Then the Picard group of the punctured spectrum of $R$ is torsion-free. If, furthermore, $R$ satisfies condition $\left(R_{2}\right)$, then the class group of $R$ is torsion-free.

Let $X$ be a complete intersection variety of dimension at least 2 in the projective space over a field. The Picard group of X modulo the hyperplane section is torsion-free.

Proof. Let $p$ be the characteristic exponent of $R$ (so it is 1 if the characteristic of $R$ is 0$)$. The fact that neither the Picard group nor $\mathrm{Cl}(R)$ has an element whose order is relatively prime to $p$ was well-known [Robbiano 1976]. Theorem 2.9 takes care of the $p$-torsion elements. The second half of the corollary follows by applying the first to the local ring at the origin of the affine cone over $X$.

Remark 2.11. The second half of the corollary was first proved in [Deligne 1973]. Another proof was given in [Bădescu 1978, Theorem B]. As far as we know, ours is the first algebraic proof. 
Example 2.12. The conditions $\operatorname{dim} R \geq 3$ and $\left(R_{2}\right)$ in the corollary cannot be weakened. Let $R=k \llbracket\left[x, y, z \rrbracket /\left(x y-z^{2}\right)\right.$, where $k$ is an algebraically closed field of characteristic other than 2 . Then $\operatorname{dim} R=2$ and $R$ is regular in codimension 1 , but $\mathrm{Cl}(R) \cong \mathbb{Z} /(2)$ (see, for example, [Fossum 1973, Proposition 11.4]).

\section{Examples of nonrigidity}

In this section we construct plenty of examples of a Gorenstein ring $R$ in positive characteristic such that $f^{n} R$ is not (strongly) rigid. This is in stark contrast to the situation for complete intersection rings, where the strong rigidity of $f^{n} R$ is known to hold. Our constructions take two completely different approaches. The first approach (see Example 3.2) provides the desired examples with torsion divisor class groups. This can be viewed as a natural consequence of Theorem 2.9. The second approach (see Example 3.5), on the contrary, provides the desired examples with torsion-free divisor class groups.

First we isolate a consequence of Theorem 2.9:

Corollary 3.1. Let $R$ be a local, Gorenstein domain with isolated singularity. Suppose that $\operatorname{dim} R \geq 3$ and $\mathrm{Cl}(R)$ has a torsion element of order $l$ that satisfies $\left(S_{3}\right)$. Then ${ }^{f^{n}} R$ is not strongly rigid for any $n$ such that $p^{n} \equiv 1$ or 0 modulo $l$. In particular, if $l=2$, then ${ }^{f^{n}} R$ is not strongly rigid for any $n$ and not rigid for $n \gg 0$.

Proof. Let $I$ be a reflexive ideal which represents an $l$-torsion element in $\mathrm{Cl}(R)$ and $q=p^{n}$. Then the ideal $J=I^{(q)}$ is isomorphic to $I$ or $R$, both of which satisfy $\left(S_{3}\right)$, contradicting Theorem 2.9. When $l=2$, for any $n, q=p^{n}$ is congruent to 0 or 1 modulo 2. The last statement follows from Example 2.2.

Example 3.2. It is not hard to find examples of isolated Gorenstein singularities with torsion class group. Let $S=k\left[x_{1}, \ldots, x_{d}\right]$ and $l$ be an integer. Let $T$ be the $l$-Veronese subring of $S$ and $R$ be the local ring at the homogeneous maximal ideal of $T$. Then one can show that $\mathrm{Cl}(R)=\mathrm{Cl}(T)=\mathbb{Z} /(l)$ using [Watanabe 1981, Theorem 1.6]. The ring $R$ obviously has an isolated singularity, as it is the local ring at the origin of the cone over a smooth projective variety. Also, $R$ will be Gorenstein as long as $l$ divides $d$. Finally, let $I$ represent the generator of $\mathrm{Cl}(T)$. It is easy to see that the cyclic cover of $T$ corresponding to $I$ is $S$, so $I$, and therefore the generator of $\mathrm{Cl}(R)$, is Cohen-Macaulay. In particular, it will be $\left(S_{3}\right)$. So all of the conditions of Corollary 3.1 can be satisfied easily.

For the rest of this section we will take another approach to construct examples of nonrigidity in which the rings have torsion-free divisor class groups. The following result gives a general technique for finding such examples:

Lemma 3.3. Let $(R, \mathfrak{m})$ be a Gorenstein ring with isolated singularity and positive dimension. The following are equivalent: 
(1) ${ }^{n} R$ is strongly rigid.

(2) For any $R$-module $L$ with infinite projective dimension, depth $F^{n}(L)=0$.

Proof. That (1) implies (2) is a consequence of Corollary 2.6. Now assume (2). Let $L$ be a module of infinite projective dimension. It is enough to prove that $\operatorname{Tor}_{1}^{R}\left(L, f^{n} R\right) \neq 0$. Consider the exact sequence

$$
0 \rightarrow L_{1} \rightarrow Q \rightarrow L \rightarrow 0
$$

where $Q$ is free and $L_{1}$ is the first syzygy of $L$. If $\operatorname{Tor}_{1}^{R}\left(L, f^{n} R\right)=0$, then by tensoring with $f^{n} R$ one gets

$$
0 \rightarrow F^{n}\left(L_{1}\right) \rightarrow Q \rightarrow F^{n}(L) \rightarrow 0 \text {. }
$$

But since $\operatorname{pd}_{R} L=\operatorname{pd}_{R} L_{1}=\infty$, one has depth $F^{n}\left(L_{1}\right)=\operatorname{depth} F^{n}(L)=0$. Since depth $Q=\operatorname{dim} Q>0$, this is a contradiction.

We also need the following crucial observation.

Lemma 3.4. Let $k$ be a field of characteristic $p>0$. Let A denote the determinantal ring $k[X] / I_{2}$ where $X=\left(X_{i j}\right)$ is a $3 \times 3$ matrix of indeterminates and $I_{2}$ is the ideal of $k[X]$ generated by all the $2 \times 2$ minors of $X$. Let $x_{i j}$ denote the images of $X_{i j}$ in $A$. Let $L=A /\left(x_{11}, x_{12}\right)$. Then depth $F^{n}(L)>0$ for all $n>0$ and $\mathrm{pd} L=\infty$.

Proof. Let $\delta_{i j}$ denote the minor of $X$ corresponding to $X_{i j}$ and $I$ be the ideal of $k[X]$ generated by $X_{11}^{n}, X_{12}^{n}$, and all the $\delta_{i j}$. We prove that for any field $k$ (we do not need to assume that $k$ has prime characteristic!) and any $n \geq 2, x_{33}$ is a nonzerodivisor for $A /\left(x_{11}^{n}, x_{12}^{n}\right) \cong k[X] / I$. In the following paragraph, we refer the reader to [Eisenbud 1995, 15.2-4] for notation and terminology (some of it italicized) regarding Gröbner bases.

We fix a reverse lexicographic order $>$ on the monomials such that

$$
X_{11}>X_{12}>X_{13}>X_{21}>X_{22}>X_{23}>X_{31}>X_{32}>X_{33} \text {. }
$$

Using Buchberger's algorithm, one can produce a Gröbner basis for $I$ consisting of all the $\delta_{i j}, X_{11}^{n}, X_{12}^{n}$, and all the monomials of the form $X_{11}^{l} X_{12}^{n-l} X_{22}^{s} X_{32}^{t}$, where $l$ runs from 1 through $n-1$ and $s, t$ run through all positive integers such that $s+t=l$. Therefore the initial ideal of $I$ (henceforth $\operatorname{in}(I))$ does not contain any monomial divisible by $X_{33}$. Assume for some $g \in k[X], X_{33} g \in I$. Let $g_{0}$ be the remainder of $g$ (with respect to the generators of $I$ ) in a standard expression obtained by performing the division algorithm. If $g_{0} \neq 0$, then $X_{33} g_{0} \neq 0$ since $k[X]$ is a domain. On the other hand, since $X_{33} g_{0} \in I$, at least one of the monomials of $X_{33} g_{0}$ is in in $(I)$. Thus, at least one of the monomials of $g_{0}$ is in in $(I)$. This contradicts the fact that $g_{0}$ is a nonzero remainder. Thus $g_{0}=0$ and $g \in I$. It follows that $x_{33}$ is a nonzerodivisor for $k[X] / I$. 
Finally, we show $\operatorname{pd} L=\infty$. Assume that $\operatorname{pd} L<\infty$, i.e., the ideal $\left(x_{11}, x_{12}\right)$ is of finite projective dimension. By [MacRae 1963, Corollary 4.4], two-generated ideals of finite projective dimension have the form $a(b, c)$, where $a$ is a nonzerodivisor and $b, c$ form a regular sequence. But if $\left(x_{11}, x_{12}\right)=a(b, c)$ for such $a, b$, and $c$, since the degree of $x_{11}$ is one, $a$ is forced to be a unit (otherwise, $\left(x_{11}, x_{12}\right)$ would be a principal ideal which is impossible). Therefore $\left(x_{11}, x_{12}\right)=(b, c)$. But since $x_{11} x_{22}-x_{21} x_{12}=0,\left(x_{11}, x_{12}\right)$ cannot be an ideal generated by a regular sequence of two elements. This is a contradiction.

Combining the two lemmas above, we get the following example. Note that the divisor class group of the ring in this example is isomorphic to $\mathbb{Z}$ [Bruns and Herzog 1993, 7.3.5], which is torsion-free.

Example 3.5. Let $R$ be the localization of the determinantal ring $A$ as in Lemma 3.4 with respect to the maximal ideal $(X)$. Then $f^{n} R$ is not strongly rigid for any $n$.

Remark 3.6. In view of the theorem of Koh and Lee mentioned in Section 1, Example 3.5 immediately yields the nonrigidity of $f^{n} R$ for any $n \geq c(R)$ (see Example 2.2). But in fact, with a little further computation, the reader can check that this example yields nonrigidity for all $n>0$ : indeed, the module $N$ of infinite projective dimension constructed in Theorem 2.5 by taking for the module $M$ the module $L$ of Lemma 3.4 satisfies $\operatorname{Tor}_{1}^{R}\left(N,{ }^{n} R\right)=0$ by the argument in the proof. But it can be shown that in fact $\operatorname{Tor}_{2}^{R}\left(N,{ }^{n} R\right) \neq 0$.

We point out that we do not know of any example showing that $f^{n} R$ is not (strongly) rigid when $\operatorname{dim} R=0$ or against a module $M$ of finite length. See, however, the discussion at the end of Section 4.

\section{Some further observations}

Throughout this section, $d$ will always be the dimension of the ring and $n$ always denotes some positive integer. We know from the previous section that $R$ could fail to be strongly rigid when $R$ is no longer a complete intersection ring. However, we still hope that to some extent such a property could hold over noncomplete intersection rings. In particular, we do not know any example showing that $f^{n} R$ is not rigid when $\operatorname{dim} R=0$ or against a module $M$ of finite length.

We first make two more definitions, the first of which is just a refinement of the definition of strong rigidity of $f^{n} R$.

Definition 4.1. Let $h$ be a nonnegative integer. ${ }^{f} R$ is called strongly rigid against modules of dimension at most $h$ if, for any integer $i$ and any finitely generated module $M$ of dimension at most $h, \operatorname{Tor}_{i}^{R}\left(M,{ }^{n} R\right)=0$ implies $\operatorname{pd}_{R} M<\infty$.

Definition 4.2. ${ }^{f^{n}} R$ is called numerically rigid if for any $R$-module $M$ of finite length, $\ell\left(F^{n}(M)\right)=p^{n d} \ell(M)$ implies $\operatorname{pd}_{R} M<\infty$. 
The latter definition is motivated by the following characterization for modules of finite projective dimension and finite length over complete intersection rings.

Theorem [Dutta 1983; Miller 2003]. Let $R$ be a complete intersection ring in characteristic $p$ and $M$ an $R$-module of finite length. Then the following are equivalent:

(1) $M$ has finite projective dimension,

(2) $\ell\left(F^{n}(M)\right)=p^{n d} \ell(M)$ for all $n>0$,

(3) $\ell\left(F^{n}(M)\right)=p^{\text {nd }} \ell(M)$ for some $n>0$.

The implication (3) $\Rightarrow(1)$ simply says that if $R$ is a complete intersection ring, then ${ }^{f^{n}} R$ is numerically rigid for any $n$. When $R$ is no longer a complete intersection ring, it is an open question whether $f^{n} R$ could still be numerically rigid. ${ }^{1}$ In fact, such a question is closely related to the rigidity question discussed earlier. The goal of this section is to explore the connections between them.

The following technical result plays a crucial role here. Recall that if $\ell(M \otimes N)$ and $\operatorname{pd} N$ are finite, then

$$
\chi(M, N) \stackrel{\operatorname{def}}{=} \sum_{j=0}^{\mathrm{pd} N}(-1)^{j} \ell\left(\operatorname{Tor}_{j}^{R}(M, N)\right) .
$$

Proposition 4.3. Let $R$ be a Noetherian local Cohen-Macaulay ring of positive dimension and of characteristic $p>0$. Let $M$ be an R-module of codimension $c$. Suppose $\operatorname{dim} M>0$ and $R_{\mathfrak{p}}$ is a complete intersection ring for every minimal prime $\mathfrak{p}$ of $M$. Then

$$
\ell\left(F_{R / x}^{n}(M / x M)\right) \geq q^{c} \chi(M, R / x)
$$

for all $n>0$ and for any system of parameters $\boldsymbol{x}$ of $F^{n}(M)$ which is also R-regular. Given $n>0$, equality holds in $(*)$ if and only if $F^{n}(M)$ is Cohen-Macaulay and $\mathrm{pd}_{R_{\mathfrak{p}}} M_{\mathfrak{p}}$ is finite for every minimal prime $\mathfrak{p}$ of $M$.

For the proof the properties of the higher Euler characteristics of Koszul complexes are used in an essential way. We recall some terms and results here.

For a pair of modules $M$ and $N$ such that $\ell(M \otimes N)<\infty$ and $\operatorname{pd} N<\infty$, the $i$-th higher Euler characteristic is defined by the formula

$$
\chi_{i}(M, N)=\sum_{j=i}^{\operatorname{pd} N}(-1)^{j-i} \ell\left(\operatorname{Tor}_{j}^{R}(M, N)\right)
$$

By convention, $\chi(M, N)=\chi_{0}(M, N)$. Some standard facts about $\chi$ and $\chi_{i}$ can be found in [Lichtenbaum 1966; Serre 1975]. In this paper, we particularly need the following two well-known results:

\footnotetext{
${ }^{1}$ The implication $(1) \Rightarrow(2)$ in the theorem fails even over Gorenstein rings [Miller and Singh 2000].
} 
Lemma 4.4 [Lichtenbaum 1966, Lemma 1]. Let $M$ be an $R$-module and $\boldsymbol{x}=$ $\left\{x_{1}, x_{2}, \ldots, x_{c}\right\}$ an $R$-sequence such that $\ell(M / x M)<\infty$. Then $\chi(M, R / \boldsymbol{x}) \geq 0$, with the equality holding if and only if $\operatorname{dim} M<c$.

Theorem 4.5 [Lichtenbaum 1966, Theorem 1]. Let $M$ be an $R$-module and $\boldsymbol{x}$ an $R$-sequence such that $\ell(M / x M)<\infty$. Then for any $i>0, \chi_{i}(M, R / x) \geq 0$, with the equality holding if and only if $\operatorname{Tor}_{i}(M, R / x)=0$ (and hence $\operatorname{Tor}_{j}(M, R / x)=0$ for all $j \geq i$ ).

Proof of Proposition 4.3. We have $\min \operatorname{Supp} F^{n}(M)=\min \operatorname{Supp} M$, since Supp $M$ and Supp $F^{n}(M)$ coincide [Peskine and Szpiro 1972]. Now write

$$
\begin{aligned}
\ell\left(F_{R / \boldsymbol{x}}^{n}(M / \boldsymbol{x} M)\right) & =\ell\left(F^{n}(M) \otimes_{R} R / \boldsymbol{x}\right) \\
& \geq \chi\left(F^{n}(M), R / \boldsymbol{x}\right) \\
& =\sum_{\mathfrak{p} \in \min \operatorname{Supp} M} \ell\left(F^{n}(M)_{\mathfrak{p}}\right) \chi(R / \mathfrak{p}, R / \boldsymbol{x}) \\
& =\sum_{\mathfrak{p} \in \min \operatorname{Supp} M} \ell\left(F_{R_{\mathfrak{p}}}^{n}\left(M_{\mathfrak{p}}\right)\right) \chi(R / \mathfrak{p}, R / \boldsymbol{x}) \\
& \geq \sum_{\mathfrak{p} \in \min \operatorname{Supp} M} q^{c} \ell\left(M_{\mathfrak{p}}\right) \chi(R / \mathfrak{p}, R / \boldsymbol{x}) \\
& =q^{c} \chi(M, R / \boldsymbol{x}),
\end{aligned}
$$

where the first inequality holds since $\chi_{1}\left(F^{n}(M), R / x\right) \geq 0$ by Theorem 4.5 , the second and last equalities hold by Lemma 4.4 , and the second inequality is a result over complete intersection rings [Dutta 1983, Theorem 1.9] (note that $R_{\mathfrak{p}}$ is complete intersection by the hypotheses).

Therefore, furthermore, equality holds if and only if $\chi_{1}\left(F^{n}(M), R / x\right)=0$ and $\ell\left(F_{R_{\mathfrak{p}}}^{n}\left(M_{\mathfrak{p}}\right)\right)=q^{c} \ell\left(M_{\mathfrak{p}}\right)$ for every minimal prime $\mathfrak{p}$ of $M$. The former is equivalent to $F^{n}(M)$ being Cohen-Macaulay by Theorem 4.5 and the latter is equivalent to $M_{\mathfrak{p}}$ having finite projective dimension over $R_{\mathfrak{p}}$ by [Miller 2003, Theorem 5.2.2], since $R_{\mathfrak{p}}$ is a complete intersection ring.

Theorem 4.6. Let $R$ be a Cohen-Macaulay local ring with isolated singularity and of positive dimension. Fix some $n>0$. If for every nonzerodivisor $y \in R$, $f^{n}(R / y R)$ is numerically rigid, then ${ }^{n} R$ is strongly rigid against modules of $d i$ mension at most one.

Proof. Let $M$ be an $R$-module of dimension at most one. Assume $f^{n}(R / y R)$ is numerically rigid for every nonzerodivisor $y \in R$. We want to prove that for any $i>$ 0 , $\operatorname{Tor}_{i}\left(M, f^{n} R\right)=0$ implies pd $M<\infty$. Let $\boldsymbol{x}=\left\{x_{1}, \ldots, x_{d-1}\right\}$ be an $R$-sequence contained in Ann $M$. We may assume that $i=1$ by replacing $M$ by its $(i-1)$-th syzygy over the ring $R /\left(x_{1}, \ldots, x_{d-1}\right)$ and using that $\operatorname{Tor}_{i}\left(R /\left(x_{1}, \ldots, x_{d-1}\right),{ }^{f^{n}} R\right)$ vanishes for all $i>0$, since $\operatorname{pd}_{R} R /\left(x_{1}, \ldots, x_{d-1}\right)<\infty$. 
Letting $K$ be the first syzygy of $M$ as an $R /\left(x_{1}, \ldots, x_{d-1}\right)$-module, we get a short exact sequence:

$$
0 \rightarrow F^{n}(K) \rightarrow F^{n}\left(\left(R /\left(x_{1}, \ldots, x_{d-1}\right)\right)^{t}\right) \rightarrow F^{n}(M) \rightarrow 0 .
$$

It follows that $F^{n}(K)$ is a Cohen-Macaulay module of dimension one. Hence by Proposition 4.3 (note that $R$ has an isolated singularity), one has $\ell\left(F_{R / y R}^{n}(K / y K)\right)=$ $q^{d-1} \chi(K, R / y R)$ for every $y \in R$ which is regular on both $K$ and $R$. Therefore, $\ell\left(F_{R / y R}^{n}(K / y K)\right)=q^{d-1} \ell(K / y K)$. Since we assume ${ }^{f^{n}}(R / y R)$ is numerically rigid, $K / y K$ has finite projective dimension over $R / y R$. Thus $K$ has finite projective dimension over $R$, whence $M$ does too by the long exact sequence of Tors against the residue field $\mathrm{k}$.

Remark 4.7. For the determinantal ring $R=k[X] / I_{2}$ used in Section 3, it was shown there that $f^{n} R$ is not strongly rigid against modules of dimension at most 5 for any $n$. In fact, we can also modify the example a little bit to show that it is not strongly rigid against modules of dimension at most 3 . For $k$ of arbitrary characteristic, though, we do not know if ${ }^{f^{n}} R$ is strongly rigid against modules of dimension at most 0,1 , or 2 . However, in characteristic 2 we have an example which shows that ${ }^{1} R$ is not strongly rigid against modules of dimension 1 . In fact, if we set $k=\mathbb{Z} / 2 \mathbb{Z}$ and take the module $N=R /\left(x_{12}, x_{13}, x_{21}, x_{23}, x_{31}, x_{32}\right)$, then it is easy to check that $\operatorname{dim} N=1$, depth $F(N)=1$, and $\operatorname{pd} N=\infty$. Taking an $R$-sequence $x_{1}, x_{2}, x_{3}, x_{4}$ contained in the annihilator of $N$ and embedding $N$ into a module of finite projective dimension over $R /\left(x_{1}, x_{2}, x_{3}, x_{4}\right)$ (via the Auslander-Buchweitz short exact sequence again), the cokernel of this embedding gives such an example. Therefore, by Theorem 4.6, we also obtain an example of a Gorenstein ring $R$ in characteristic 2 for which the corresponding $R$-module ${ }^{f^{1}} R$ is not numerically rigid.

Acknowledgements. We thank Craig Huneke for crucial remarks which connected our works, and Srikanth Iyengar, who carefully read an earlier version of this manuscript and suggested a better statement for Corollary 2.6. We also thank the anonymous referee for many helpful comments.

\section{References}

[Auslander and Buchweitz 1989] M. Auslander and R.-O. Buchweitz, "The homological theory of maximal Cohen-Macaulay approximations”, pp. 5-37 Mém. Soc. Math. France (N.S.) 38, Soc. Math. de France, Paris, 1989. MR 91h:13010 Zbl 0697.13005

[Avramov and Miller 2001] L. L. Avramov and C. Miller, "Frobenius powers of complete intersections”, Math. Res. Lett. 8:1-2 (2001), 225-232. MR 2002b:13022 Zbl 1034.13006

[Avramov et al. 2006] L. L. Avramov, S. Iyengar, and C. Miller, "Homology over local homomorphisms”, Amer. J. Math. 128:1 (2006), 23-90. MR 2007e:13027 Zbl 1102.13011

[Bădescu 1978] L. Bădescu, "A remark on the Grothendieck-Lefschetz theorem about the Picard group”, Nagoya Math. J. 71 (1978), 169-179. MR 80g:14012 Zbl 0358.14026 
[Bourbaki 1965] N. Bourbaki, Algèbre commutative, ch. 7: diviseurs, Actualités Scientifiques et Industrielles 1314, Hermann, Paris, 1965. Translated as part of Commutative algebra, Hermann, Paris, 1972. MR 41 \#5339

[Braun 2004] A. Braun, "On a question of M. Auslander", J. Algebra 276:2 (2004), 674-684. MR 2005a:13020 Zbl 1084.13004

[Bruns and Herzog 1993] W. Bruns and J. Herzog, Cohen-Macaulay rings, Cambridge Studies in Advanced Mathematics 39, Cambridge University Press, 1993. MR 95h:13020 Zbl 0788.13005

[Dao 2010] H. L. Dao, "Decency and Tor-rigidity for modules over hypersurfaces", preprint, 2010. arXiv math/0611568v3

[Deligne 1973] P. Deligne, "Cohomologie des intersections completes", pp. 39-61 in SGA 7 XI (Séminaire de Géométrie Algébrique du Bois Marie 1967-1969), Lect. Notes Math. 340, Springer, Berlin, 1973. Zbl 0265.14007

[Dutta 1983] S. P. Dutta, "Frobenius and multiplicities", J. Algebra 85:2 (1983), 424-448. MR 85f: 13022 Zbl 0527.13014

[Dutta 2003] S. P. Dutta, "On modules of finite projective dimension over complete intersections", Proc. Amer. Math. Soc. 131:1 (2003), 113-116. MR 2003j:13016 Zbl 1007.13006

[Eisenbud 1995] D. Eisenbud, Commutative algebra, Graduate Texts in Mathematics 150, Springer, New York, 1995. MR 97a:13001 Zbl 0819.13001

[Fossum 1973] R. M. Fossum, The divisor class group of a Krull domain, Ergebnisse der Math. 74, Springer, New York, 1973. MR 52 \#3139 Zbl 0256.13001

[Gabber 2004] O. Gabber, "On purity for the Brauer group”, pp. 1975-1977 in Arithmetic Algebraic Geometry, Oberwolfach Report 37, 2004.

[Herzog 1974] J. Herzog, "Ringe der Charakteristik $p$ und Frobeniusfunktoren", Math. Z. 140 (1974), 67-78. MR 50 \#4569 Zbl 0278.13006

[Iyengar and Sather-Wagstaff 2004] S. Iyengar and S. Sather-Wagstaff, "G-dimension over local homomorphisms: applications to the Frobenius endomorphism", Illinois J. Math. 48:1 (2004), 241272. MR 2005c:13016 Zbl 1103.13009

[Koh and Lee 1998] J. Koh and K. Lee, "Some restrictions on the maps in minimal resolutions", $J$. Algebra 202:2 (1998), 671-689. MR 99d:13017 Zbl 0909.13008

[Kunz 1969] E. Kunz, "Characterizations of regular local rings for characteristic p", Amer. J. Math. 91 (1969), 772-784. MR 40 \#5609 Zbl 0188.33702

[Lichtenbaum 1966] S. Lichtenbaum, "On the vanishing of Tor in regular local rings", Illinois J. Math. 10 (1966), 220-226. MR 32 \#5688 Zbl 0139.26601

[MacRae 1963] R. E. MacRae, "On the homological dimension of certain ideals", Proc. Amer. Math. Soc. 14 (1963), 746-750. MR 28 \#5103

[Miller 2003] C. Miller, “The Frobenius endomorphism and homological dimensions”, pp. 207-234 in Commutative algebra (Grenoble/Lyon, 2001), edited by L. L. Avramov et al., Contemp. Math. 331, Amer. Math. Soc., Providence, RI, 2003. MR 2004j:13020 Zbl 1085.13502

[Miller and Singh 2000] C. M. Miller and A. K. Singh, "Intersection multiplicities over Gorenstein rings", Math. Ann. 317:1 (2000), 155-171. MR 2001h:13031 Zbl 0970.13007

[Peskine and Szpiro 1969] C. Peskine and L. Szpiro, "Sur la topologie des sous-schémas fermés d'un shéma localement noethérien, définis comme support d'un faisceau cohérent localement de dimension projective finie", C. R. Acad. Sci. Paris Sér. A 269 (1969), 49-51. MR 40 \#1385 Zbl 0184.29001 
On the (non)rigidity of the Frobenius endomorphism over Gorenstein rings 1053

[Peskine and Szpiro 1972] C. Peskine and L. Szpiro, "Dimension projective finie et cohomologie locale. Applications à la démonstration de conjectures de M. Auslander, H. Bass et A. Grothendieck", Inst. Hautes Études Sci. Publ. Math. 42 (1972), 47-119. MR 51 \#10330 Zbl 0268.13008

[Robbiano 1976] L. Robbiano, "Some properties of complete intersections in "good" projective varieties”, Nagoya Math. J. 61 (1976), 103-111. MR 53 \#13222 Zbl 0309.14043

[Serre 1975] J.-P. Serre, Algèbre locale: multiplicités, 3e ed., Lecture Notes in Math. 11, Springer, Berlin, 1975. Zbl 0296.13018

[Watanabe 1981] K. Watanabe, "Some remarks concerning Demazure's construction of normal graded rings", Nagoya Math. J. 83 (1981), 203-211. MR 83g:13016 Zbl 0518.13003

Communicated by Kei-Ichi Watanabe

Received 2009-09-11 Revised 2010-05-09 Accepted 2010-06-11

hdao@math.ku.edu

Department of Mathematics, University of Kansas, Lawrence, KS 66045-7523, United States

jinjia.li@louisville.edu

Department of Mathematical Sciences, Middle Tennessee State University, Murfreesboro, TN 37132, United States

Current address:

Department of Mathematics, University of Louisville, 328 Natural Sciences Building, Louisville, KY 40292, United States

clamille@syr.edu

Mathematics Department, Syracuse University, Syracuse, NY 13244-1150, United States 


\section{Algebra \& Number Theory}

www.jant.org

\section{EDITORS}

\section{MANAGING EDITOR}

Bjorn Poonen

Massachusetts Institute of Technology

Cambridge, USA

\author{
EDITORIAL BOARD CHAIR \\ David Eisenbud \\ University of California \\ Berkeley, USA
}

\section{BOARD OF EDITORS}

\section{Georgia Benkart}

Dave Benson

Richard E. Borcherds

John H. Coates

J-L. Colliot-Thélène

Brian D. Conrad

Hélène Esnault

Hubert Flenner

Edward Frenkel

Andrew Granville

Joseph Gubeladze

Ehud Hrushovski

Craig Huneke

Mikhail Kapranov

Yujiro Kawamata

János Kollár

Hendrik W. Lenstra

Yuri Manin

Barry Mazur
University of Wisconsin, Madison, USA

University of Aberdeen, Scotland

University of California, Berkeley, USA

University of Cambridge, UK

CNRS, Université Paris-Sud, France

University of Michigan, USA

Universität Duisburg-Essen, Germany

Ruhr-Universität, Germany

University of California, Berkeley, USA

Université de Montréal, Canada

San Francisco State University, USA

Hebrew University, Israel

University of Kansas, USA

Yale University, USA

University of Tokyo, Japan

Princeton University, USA

Universiteit Leiden, The Netherlands

Northwestern University, USA

Harvard University, USA
Susan Montgomery

Shigefumi Mori

Andrei Okounkov

Raman Parimala

Victor Reiner

Karl Rubin

Peter Sarnak

Michael Singer

Ronald Solomon

Vasudevan Srinivas

J. Toby Stafford

Bernd Sturmfels

Richard Taylor

Ravi Vakil

Michel van den Bergh

Marie-France Vignéras

Kei-Ichi Watanabe

Andrei Zelevinsky

Efim Zelmanov
University of Southern California, USA

RIMS, Kyoto University, Japan

Princeton University, USA

Emory University, USA

University of Minnesota, USA

University of California, Irvine, USA

Princeton University, USA

North Carolina State University, USA

Ohio State University, USA

Tata Inst. of Fund. Research, India

University of Michigan, USA

University of California, Berkeley, USA

Harvard University, USA

Stanford University, USA

Hasselt University, Belgium

Université Paris VII, France

Nihon University, Japan

Northeastern University, USA

University of California, San Diego, USA

\section{PRODUCTION}

ant@mathscipub.org

Silvio Levy, Scientific Editor

Andrew Levy, Production Editor

See inside back cover or www.jant.org for submission instructions.

The subscription price for 2010 is US \$140/year for the electronic version, and \$200/year (+\$30 shipping outside the US) for print and electronic. Subscriptions, requests for back issues from the last three years and changes of subscribers address should be sent to Mathematical Sciences Publishers, Department of Mathematics, University of California, Berkeley, CA 94720-3840, USA.

Algebra \& Number Theory (ISSN 1937-0652) at Mathematical Sciences Publishers, Department of Mathematics, University of California, Berkeley, CA 94720-3840 is published continuously online. Periodical rate postage paid at Berkeley, CA 94704, and additional mailing offices.

ANT peer review and production are managed by EditFLOW ${ }^{\mathrm{TM}}$ from Mathematical Sciences Publishers.

\section{PUBLISHED BY}

mathematical sciences publishers

http://www.mathscipub.org

A NON-PROFIT CORPORATION

Typeset in LATEX

Copyright $\odot 2010$ by Mathematical Sciences Publishers 


\section{Algebra \& Number Theory}

Volume $4 \quad$ No. $8 \quad 2010$

On ramification filtrations and $p$-adic differential modules, I: the equal characteristic case 969 LIANG XIAO

Exponential generation and largeness for compact $p$-adic Lie groups

MICHAEL LARSEN

On the (non)rigidity of the Frobenius endomorphism over Gorenstein rings

Hailong DaO, JinJia Li and Claudia Miller

A lower bound on the essential dimension of simple algebras

ALEXANDER S. MERKURJEV

On the minimal ramification problem for semiabelian groups

HERShy KisILEVSKY, DANNy NEFTIN and JACK SONN

Remarks on modular symbols for Maass wave forms

YURI I. MANIN 